\title{
Mutation analysis by direct and whole exome sequencing in familial and sporadic tooth agenesis
}

\author{
ALESSANDRO SALVI $^{1}$, EDOARDO GIACOPUZZI ${ }^{1}$, ELENA BARDELLINI ${ }^{2}$, FRANCESCA AMADORI ${ }^{2}$, \\ LIA FERRARI $^{1}$, GIUSEPPINA DE PETRO ${ }^{1}$, GIUSEPPE BORSANI ${ }^{1}$ and ALESSANDRA MAJORANA ${ }^{2}$ \\ ${ }^{1}$ Department of Molecular and Translational Medicine, Division of Biology and Genetics, University of Brescia; \\ ${ }^{2}$ Department of Medical and Surgical Specialties, Radiological Sciences and Public Health, \\ Dental Clinic, University of Brescia, I-25123 Brescia, Italy
}

Received April 13, 2016; Accepted July 25, 2016

DOI: $10.3892 / \mathrm{ijmm} .2016 .2742$

\begin{abstract}
Dental agenesis is one of the most common congenital craniofacial abnormalities. Dental agenesis can be classified, relative to the number of missing teeth (excluding third molars), as hypodontia (1 to 5 missing teeth), oligodontia (6 or more missing teeth), or anodontia (lack of all teeth). Tooth agenesis may occur either in association with genetic syndromes, based on the presence of other inherited abnormalities, or as a non-syndromic trait, with both familiar and sporadic cases reported. In this study, we enrolled 16 individuals affected by tooth agenesis, prevalently hypodontia, and we carried out direct Sanger sequencing of paired box 9 (PAX9) and Msh homeobox 1 (MSX1) genes in 9 subjects. Since no mutations were identified, we performed whole exome sequencing (WES) in the members of 5 families to identify causative gene mutations either novel or previously described. Three individuals carried a known homozygous disease mutation in the Wnt family member 10A (WNT10A) gene (rs121908120). Interestingly, two of these individuals were siblings and also carried a heterozygous functional variant in EDAR-associated death domain (EDARADD) (rs114632254), another disease causing gene, generating a combination of genetic variants never described until now. The analysis of exome sequencing data in the members of other 3 families highlighted new candidate genes potentially involved in tooth agenesis and considered suitable for future studies. Overall, our study confirmed the major role played by WNT10A in tooth agenesis and the genetic heterogeneity of this disease. Moreover, as more genes are shown to be involved in tooth
\end{abstract}

Correspondence to: Dr Alessandro Salvi or Professor Giuseppina De Petro, Department of Molecular and Translational Medicine, Division of Biology and Genetics, University of Brescia, Viale Europa 11, I-25123 Brescia, Italy

E-mail: alessandro.salvi@unibs.it

E-mail: giuseppina.depetro@unibs.it

Key words: hypodontia, oligodontia, PAX9, MSX1, DNA mutational analysis, point mutation agenesis, WES analysis may be an effective approach to search for genetic variants in familiar or sporadic tooth agenesis, at least in more severe clinical manifestations.

\section{Introduction}

Dental agenesis is one of the most common congenital anomaly of human dentition, with a prevalence (excluding third molars) ranging from 2.6 to $11.3 \%$ (1). Relative to the number of missing teeth, dental agenesis can be classified as hypodontia (when 1 to 5 teeth are missing), oligodontia (when 6 or more teeth are missing), or anodontia (when the complete failure of dentition development occurs). The most frequently missing teeth are the mandibular second premolars, followed by the maxillary lateral incisors and maxillary second premolars (2). It is generally accepted that both genetic and environmental factors play a significant role in the pathogenesis of this disease (3). Tooth agenesis may occur either in association with genetic syndromes characterized by other inherited anomalies as an isolated nonsyndromic familial trait, or as sporadic cases. Familial tooth agenesis is characterized by moderate genetic heterogeneity and has been reported to have an autosomal-dominant, autosomalrecessive or X-linked mode of inheritance $(4,5)$.

Tooth development requires a complex network of molecular interactions in which the activation of the nuclear factor- $\kappa \mathrm{B}(\mathrm{NF}-\kappa \mathrm{B})$ and WNT signaling pathways indeed play a central role. The transcription factor, $N F-\kappa B$, controls several cellular functions and it is activated by members of the tumor necrosis factor receptor (TNFR) superfamily, including EDA receptor (EDAR). Ectodysplasin (EDA) is a member of the tumor necrosis factor (TNF)-related ligand family and binds to its membrane receptor, EDAR, that in turn binds to its adaptor, EDAR-associated death domain (EDARADD). The EDA-EDAR-EDARADD complex activates the NEMO-IKK signaling cascade, allowing the nuclear translocation of NF- $\mathrm{B}$ and thus the transcriptional control of genes that are crucial for tooth development (6).

WNT signaling molecules play important roles in the differentiation of tissues and organs during embryonic development. The secretion of WNTs, including WNT4, WNT6, and WNT10 from the dental epithelium is essential for tooth development. WNT10A is expressed in the dental epithelium 
and mesenchyme. Similar to other WNT proteins, it binds to the Frizzled $(\mathrm{Fz})$ transmembrane receptors and to lipoprotein receptor-related protein 5/6 (LRP5/6) coreceptors, leading to the activation of the $\beta$-catenin pathway (7).

One of the direct downstream targets of $\mathrm{WNT} / \beta$ catenin signaling during craniofacial development is Msh homeobox 1 (MSX1). MSX1 is a homeobox gene encoding a transcription factor that regulates the expression of bone morphogenetic protein 4 (BMP4), a member of the transforming growth factor- $\beta$ (TGF- $\beta$ ) superfamily, during the bud and cap stages of the tooth development. In these phases, MSX1 interacts with paired box 9 (PAX9) both at the gene and protein levels, with MSX1 and PAX9 being intimately involved in the genetic networks regulating tooth development (8). PAX9 is a transcriptional factor that plays a key role during embryogenesis by modifying the transcriptional activity of downstream genes. In particular, by physically associating with MSX1, it enhances both MSX1 and BMP4 gene expression during tooth development $(9,10)$. On the other hand, Axin 2 (AXIN2), also known as conductin/axil, is an inhibitor of the WNT signaling pathway (11).

Previously, dominant loss-of-function mutations in MSX1, PAX9 and AXIN2 have been described in familial forms of non-syndromic tooth agenesis (12). Recently, the WNT10A variant has been described in up to $50 \%$ of analyzed patients with dental agenesis and also in various ectodermal dysplasia syndromes $(13,14)$. Mutations in EDA, EDAR and EDARADD have also been shown to be associated with both isolated tooth agenesis and syndromic tooth agenesis, such as X-linked hypohidrotic ectodermal dysplasia (XLHED), a rare anomaly involving sparse hair, dental abnormalities, skin lesions and hypoplasia of sweat glands (15).

The initial aim of this study was to evaluate the MSX1 and PAX9 mutation rate in a cohort of patients and subsequently to identify the causative gene mutations associated with autosomal tooth agenesis in some families by employing whole exome sequencing (WES).

\section{Subjects and methods}

Study subjects. The probands ( $\mathrm{n}=9$; Fig. 1) were patients who were 10 to 20 years of age enrolled at the Dental Clinic of the University of Brescia, Italy, from 2008 to 2014, with a confirmed diagnosis of dental agenesis, but without systemic or syndromic diseases. When available, parents and siblings either affected or not, were also enrolled in the study. All individuals enrolled were informed of the purpose of this study and signed an informed consent. All clinical and genetic studies were approved by the Ethics Committee of the Spedali Civili of Brescia (AOBS-GENI-2011; NP 1119) and were conducted according to the principles expressed in the Declaration of Helsinki. In addition to panoramic radiography, a clinical examination was conducted on a dental chair, under artificial light, with a probe and a dental mirror by two trained dentists.

Mutation analysis by Sanger sequencing. Genomic DNA was isolated from the peripheral blood of affected individuals and available affected/non-affected relatives using the Purgene Blood Core kit (Qiagen, Valencia, CA, USA) following the instructions of the manufacturer. DNA from peripheral blood of a control (CTRL) unaffected subject was also isolated. The
PAX9 and MSX1 coding sequences were amplified by PCR using the primers and the thermal cycler protocols previously described (16-18). The amplified products were sequenced using the BigDye Terminator v3.1 Cycle Sequencing kit and analyzed using the ABI PRISM 310xl Genetic Analyzer (both from Applied Biosystems, Foster City, CA, USA). The results were compared with the reference sequences for each (MSX1, NM_002448.3; PAX9, NM_006194.3).

The exons and approximately $30 \mathrm{bp}$ of the flanking introns of the WNT10A and EDARADD genes were amplified with the primers previously described (19). The results were compared with the reference sequences for each gene (WNT10A, NM_025216.2; EDARADD, NM_080738.3).

Whole exome sequencing (WES). WES was carried out in selected affected individuals and their relatives. In total, 12 cases were examined by WES and the details of the subjects analyzed are reported in Fig. 1. The exomes of the probands of families 1, 5, 7 and 8 were sequenced at Personal Genomics (University of Verona, Italy) using the TruSeq Exome Enrichment kit (Illumina, San Diego, CA, USA) for whole exome capture. The members of family 5 (I-1, I-2, II-2), family 8 (I-1, I-2) and family 9 (I-2, II-2, II-3) had their exomes sequenced at Erasmus MC, University Medical Center Rotterdam using the Nimblegen SeqCap EZ Exome version 2.0 exome capture kit. In both protocols, paired-end libraries were sequenced on the Illumina HiSeq instrument. Sequencing reads were aligned to the human genome reference sequence (hg19) using BWA-mem (20) and then processed according to GATK 3.4-46 best practices (21) for variant discovery. Briefly, aligned reads were processed for duplicate removal using Picard and the base quality score recalibration was performed using GATK. The processed BAM files of single probands or the proband and relatives were then analyzed using GATK HaplotypeCaller for the identification of both single nucleotide polymorphisms (SNPs) and indel variants. Reported variants in VCF file format were then filtered removing those with a read depth $<6$ and a genotype quality (GQ) $<20$. Subsequently, the filtered variants were annotated using ANNOVAR (22) and selected according to the following criteria: i) exonic, non-synonymous variants; ii) reported allele frequency in 1000G phase 3 data and ExAc $0.3<1 \%$; and iii) not located in segmental duplicated region. These variants were searched for mutations in known genes related to dental agenesis. Where no mutations emerged, the data of the family pedigree were analyzed to select variants segregating according to the disease hereditary model. These variants were further annotated with known phenotypes from the ClinVar database (23) and the RVIS score (24) that provides an estimate of the tolerance to functional variations for each gene. Candidate genes variants were then prioritized based on PolyPhen2 (25) and FATHMM (26) deleteriousness predictions and the RVIS score of the associated gene.

\section{Results}

Study subjects. A total of 9 females and 7 males ranging in age from 8 to 48 years affected by tooth agenesis from 9 different families were enrolled in this study (Fig. 1). In particular, 5 individuals were affected by oligodontia (number of missing teeth $\geq 6$ ) and 11 individuals by hypodontia (number 

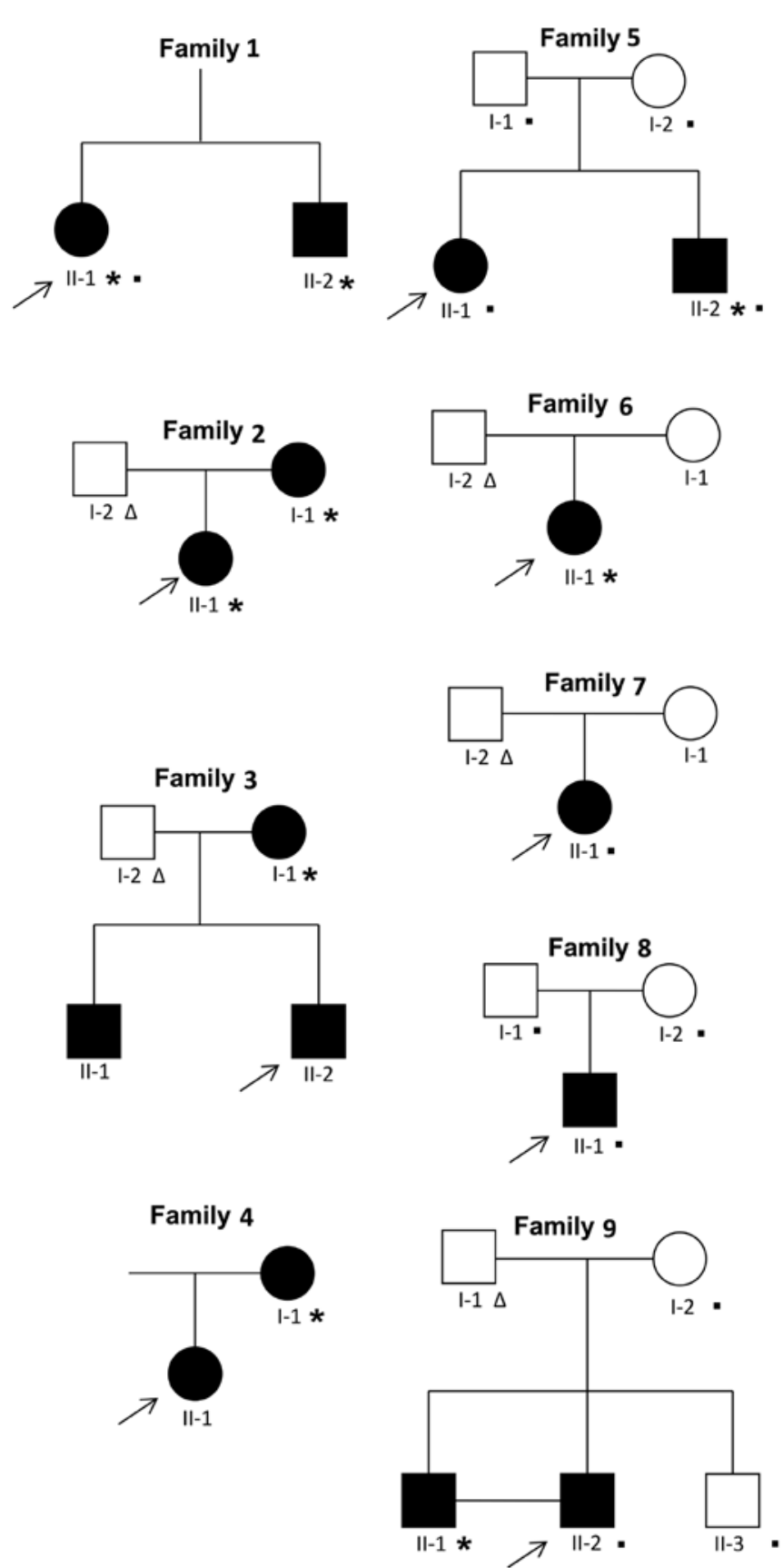

Figure 1. Pedigrees of the 9 families in this study. Squares indicaate males, and circles indicate females. Filled symbols represent individuals diagnosed with tooth agenesis; empty symbols represent unaffected subjects. The asterisks indicate individuals for which mutational analysis for the PAX9 and MSX1 genes was performed; filled squares indicate subjects who underwent whole exome sequencing (WES) analysis; empty triangles indicate that no DNA was available. Arrows indicate the probands of each family.

of missing teeth $\leq 5)$. The most common missing teeth were the mandibular second premolars ( 35 in 10/16 affected; 45 in 9/16 affected), maxillary lateral incisors (12 in 5/16 affected; 22 in 5/16 affected) and maxillary second premolars (15 in 4/16 affected; 25 in 4/16 affected) (Tables I and II).

Mutation analysis of PAX9 and MSX1 genes. To identify causative mutations in subjects with tooth agenesis, we performed direct sequencing of the PAX9 and MSX1 genes in 9 affected subjects (Fig. 1, individuals marked with an asterisk).
Table I. Characteristics of the probands and the affected relatives.

\begin{tabular}{lrr}
\hline Characteristic & $\mathrm{N}$ & $\%$ \\
\hline Males & 7 & 44 \\
Females & 9 & 56 \\
Type of tooth agenesis (total number of cases) & 16 & \\
Hypodontia (1-5 permanent teeth missing) & 11 & 69 \\
Oligodontia (>6 permanent teeth missing) & 5 & 31 \\
Type of permanent teeth missing & 81 & \\
(total teeth missing) & & \\
Central incisor $(11,21,31,41)$ & 8 & 9.9 \\
Lateral incisor $(12,22,32,42)$ & 16 & 19.7 \\
Canine $(13,23,33,43)$ & 4 & 4.9 \\
First premolar $(14,24,34,44)$ & 9 & 11.1 \\
Second premolar $(15,25,35,45)$ & 27 & 33.3 \\
First molar $(16,26,36,46)$ & 1 & 1.2 \\
Second molar $(17,27,37,47)$ & 6 & 7.4 \\
Third molar $(18,28,38,48)$ & 10 & 12.3 \\
No. of missing incisors & 35 & 43.2 \\
No. of missing canines & 4 & 4.9 \\
No. of missing premolars & 25 & 30.8 \\
No. of missing molars & 17 & 21 \\
\hline & &
\end{tabular}

Ultimately, the mutation analysis of the PAX9 and MSX1 genes did not highlight the presence of any causative mutation in the 9 affected individuals.

WES. Since the genetic heterogeneity of tooth agenesis has become apparent and has expanded since our first screening, to include 17 different genes, we decided to apply WES to identify novel and/or previously described causative mutations, as well as new candidate genes. Overall, we performed WES on 12 subjects from 5 distinct families (Fig. 1, individuals marked with a filled square; Table II, individuals highlighted in grey) with a mean coverage across target region of $75 \mathrm{X}-171 \mathrm{X}$ and $22,482-37,874$ variants identified per subject in exonic regions (Table III).

For 2 families, it was possible to identify known causative mutations, thus providing a molecular diagnosis. The already described p.F228I mutation (rs121908120) in the homozygous state in the WNT10A gene was detected in the proband of family 7 and confirmed by Sanger sequencing; her unaffected mother displayed the same variant in the heterozygous state (Fig. 2). The same mutation was also detected in both the affected siblings from family 1 (Fig. 3). Moreover, the proband (II-1, oligodontia) and her brother (II-2, more severe phenotype) also carried a p.S103F mutation (rs114632254) in the EDARADD gene in the heterozygous state (Fig. 3). Both mutations were confirmed by Sanger sequencing. In 3 other families, our analysis led to the identification of novel candidate variants in genes with a potential role in tooth agenesis.

In family 5 , an autosomal recessive form of inheritance seemed to be most probable (Fig. 1). In both the affected 


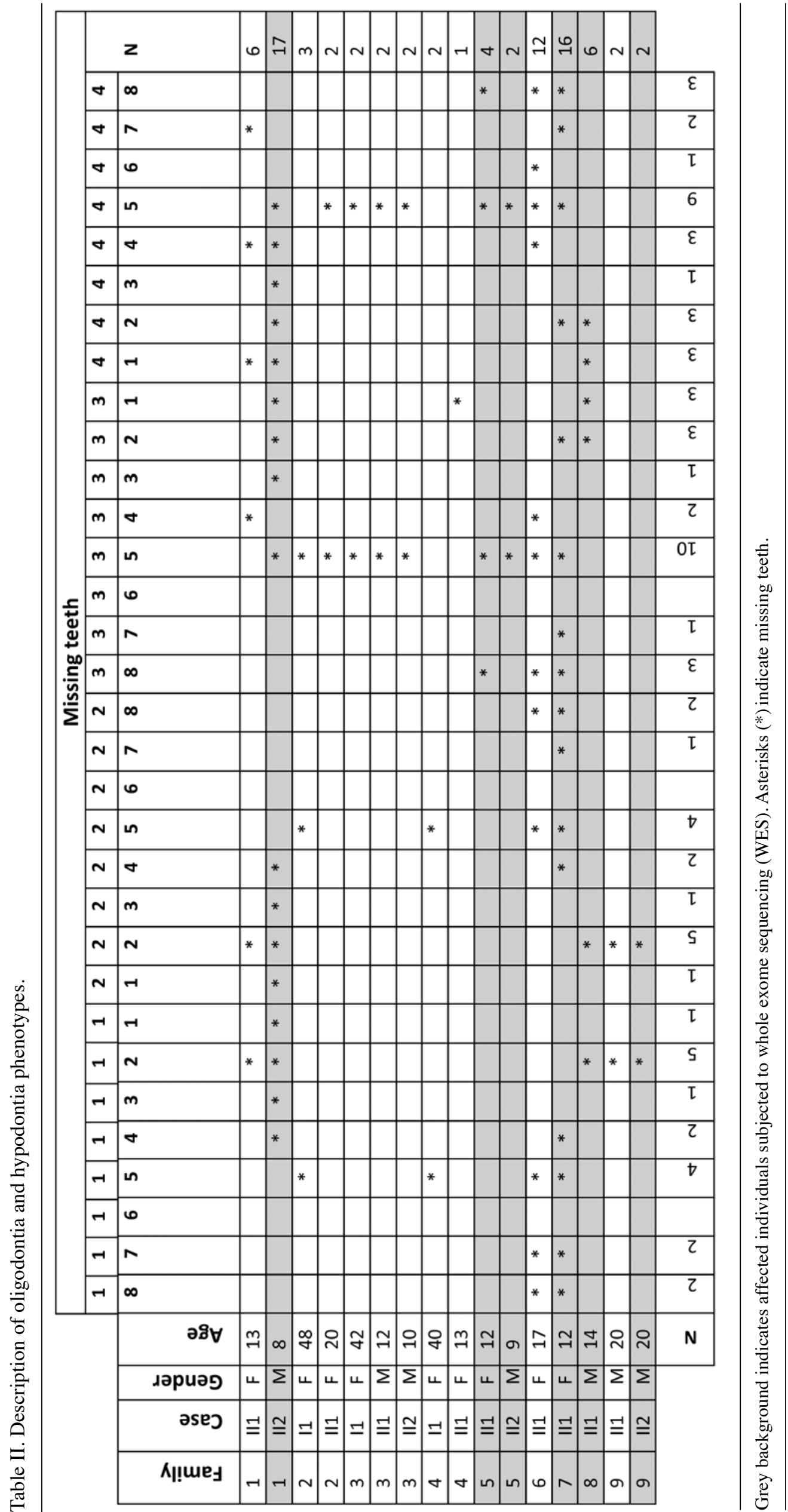




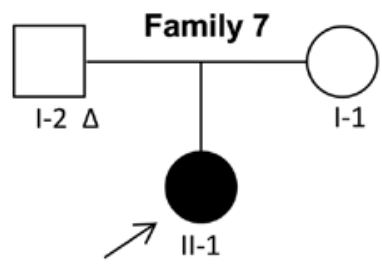

WNT10A c.T682A; p.F228I hom

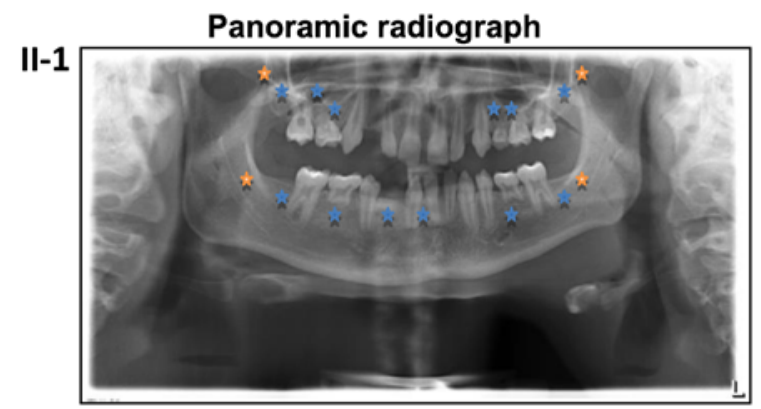

WNT10A p.F228I/ p.F228I

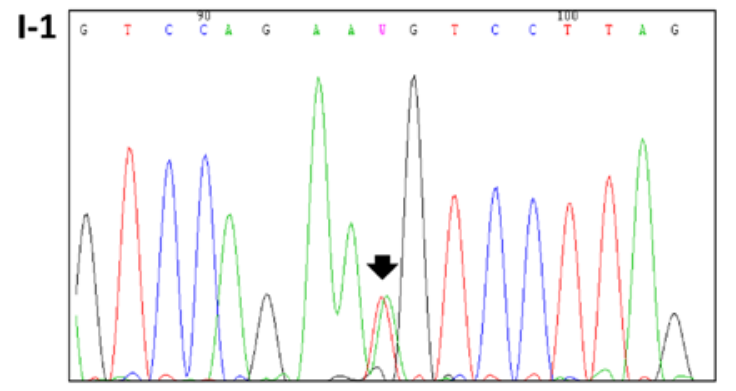

II-1

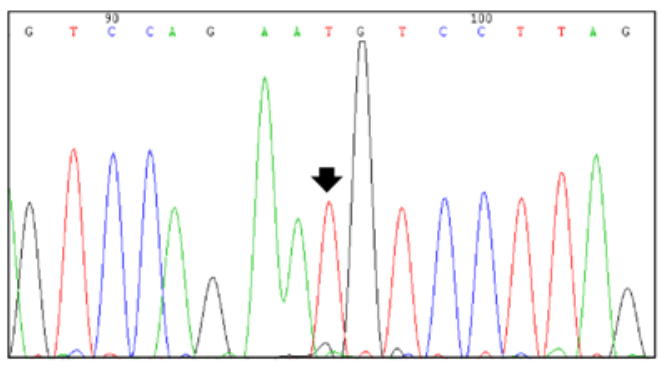

CTRL

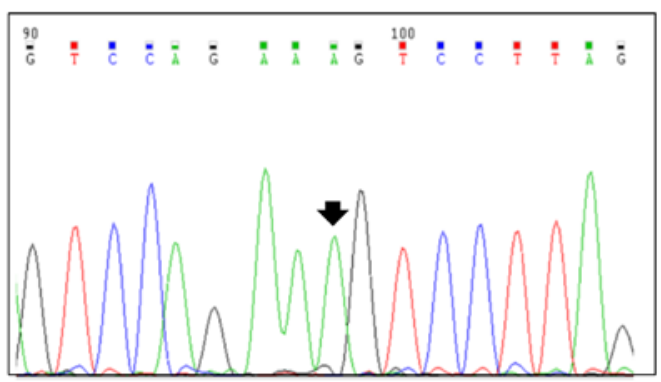

Figure 2. Panoramic radiograph of dentition of the proband (II-1) of family 7. The missing teeth are indicated by asterisks. Partial DNA sequences of exon 3 of WNT10A from the unaffected mother (subject I-1), the proband (II-1) and from a control subject (CTRL). The nucleotide substitution is indicated by an arrow.

siblings, we found compound heterozygous mutations in the Mucin 16 (MUC16) and Titin (TTN) genes and a homozygous genetic variation c.-387delC/G in the 5 'UTR portion of the paired-like homeodomain 2 (PITX2) gene. Furthermore, in the proband (II-1) and in her brother, we detected an SNP (c. T455C:p.V152A; rs17563) in the homozygous state in the BMP4 gene previously associated with tooth agenesis (Table IV).
Table III. Summary of whole exome sequencing results.

\begin{tabular}{lcccc}
\hline Sample & Family & $\begin{array}{c}\text { Mean } \\
\text { coverage in } \\
\text { target region }\end{array}$ & $\begin{array}{c}\text { \% bases } \\
\text { covered at } \\
\text { least 20X }\end{array}$ & $\begin{array}{c}\text { No. of } \\
\text { exonic } \\
\text { variants }\end{array}$ \\
\hline II-1 & 5 & $100 \mathrm{X}$ & 93.6 & 23,297 \\
II-1 & 1 & $171 \mathrm{X}$ & 95.5 & 22,801 \\
II-1 & 7 & $77 \mathrm{X}$ & 91.8 & 22,482 \\
II-1 & 8 & $82 \mathrm{X}$ & 92.2 & 23,564 \\
I-1 & 5 & $75 \mathrm{X}$ & 83.9 & 22,603 \\
II-2 & 5 & $82 \mathrm{X}$ & 85.3 & 22,822 \\
I-2 & 5 & $82 \mathrm{X}$ & 85.3 & 22,706 \\
I-1 & 8 & $83 \mathrm{X}$ & 85.8 & 22,825 \\
I-2 & 8 & $80 \mathrm{X}$ & 84.9 & 23,129 \\
I-2 & 9 & $82 \mathrm{X}$ & 91.6 & 37,874 \\
II-2 & 9 & $100 \mathrm{X}$ & 92.7 & 37,822 \\
II-3 & 9 & $88 \mathrm{X}$ & 91.1 & 37,556 \\
\hline
\end{tabular}

All results are reported considering the human CDS sequences as target region. Family ID are the same as those reported in Fig. 1.

Family 8 was also likely to present a recessive form of inheritance (Fig. 1). The proband displayed homozygous non-synonymous single nucleotide variants (SNVs) in the arylsulfatase family member H (ARSH), proline rich 32 (PRR32) and apurinic/apyrimidinic endodeoxyribonuclease 2 (APEX2) genes, a nucleotide change in homozygosis in transglutaminase 4 (TGM4) resulting in a premature stop codon and a $40 \mathrm{bp}$ frameshift insertion in homozygosis in the placenta specific 4 (PLAC4) gene. Putative de novo heterozygous genetic variants were identified in 14 genes, 4 of whom displayed compound heterozygous gene alterations. Furthermore, the homozygous genetic variant in the 5'UTR portion of the PITX2 gene identified in family 6 was also present in the proband of family 8 (Table V).

Finally, an autosomal recessive form of inheritance was likely also for family 9 . The father could not be included in the study and the analysis was conducted on the twin proband (II-2) and on his unaffected mother and brother (Fig. 1). The proband evidenced a non-synonymous SNP in the heterozygous state in the ankyrin repeat and EF-hand domain containing 1 (ANKEF1), COMM domain-containing protein 7 (COMMD7), DDHD domain containing 1 (DDHD1), early B-cell factor 2 (EBF2), envoplakin (EVPL), SPT6 homolog, histone chaperone (SUPT6H) genes and a compound heterozygous mutation in the succinate-CoA ligase GDP-forming beta subunit (SUCLG2) gene (Table VI).

\section{Discussion}

The main objective of this study was to identify the causative genetic defects in subjects affected by non-syndromic tooth agenesis. We began with Sanger sequencing of the PAX9 and MSX1 genes, since they were considered in the literature the most probable candidate genes (27). This analysis performed on 9 individuals affected by agenesis did not evidence any muta- 


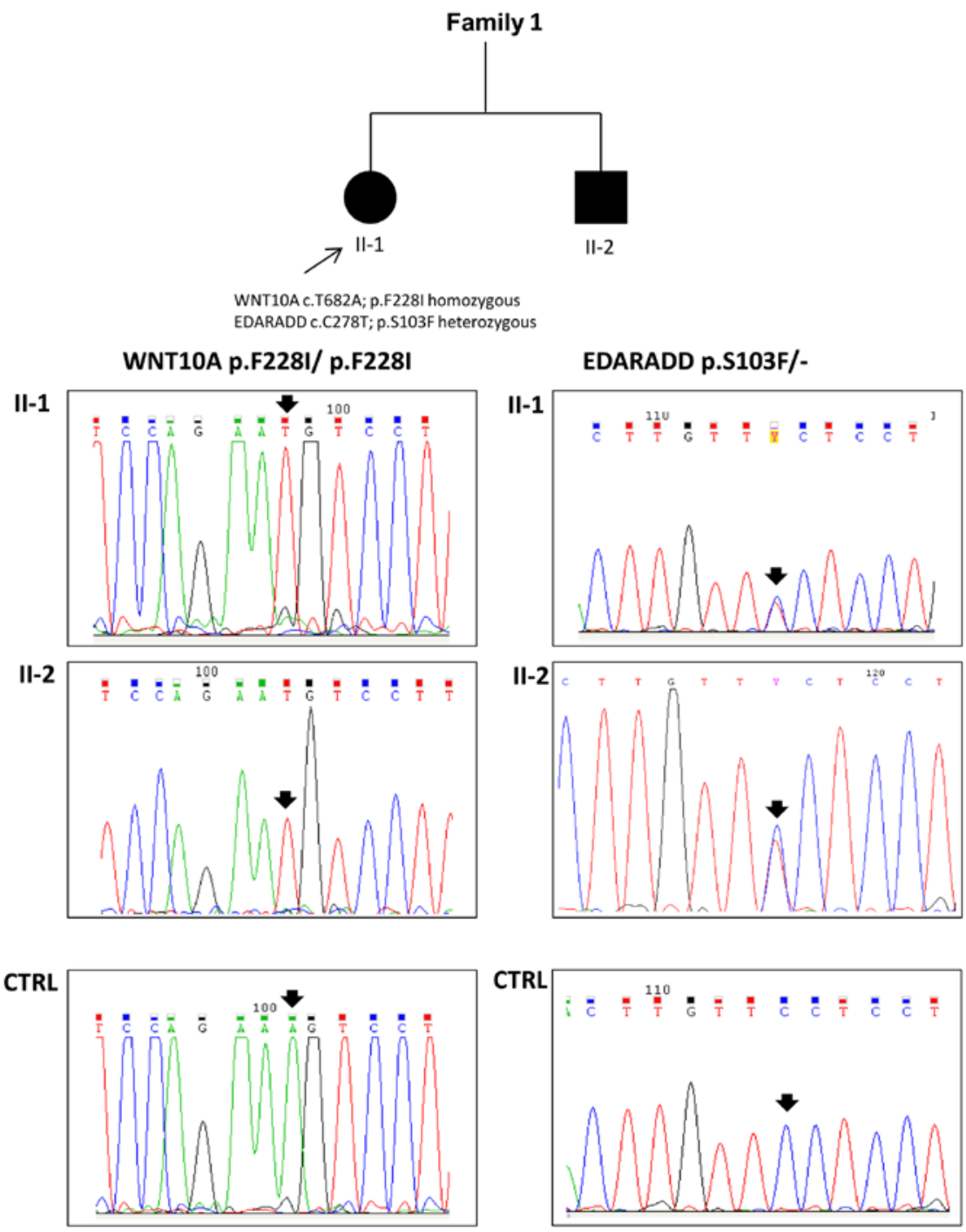

Figure 3. Direct sequencing analysis of mutation found by whole exome sequencing (WES) in family 1. Partial DNA sequences of exon 3 of WNT10A (left panels) and exon 7 of EDARADD (right panels) from the proband (II-1), her brother (II-2) and from a control subject. Nucleotide substitution is indicated by an arrow.

tion, thus suggesting that other genes may be involved in tooth agenesis, in agreement with other published data (28) Mutations in the WNT10A gene emerged as frequently involved $(13,14)$ We decided to use the WES technology to identify causative mutations. We identified pathogenic mutations in the proband of family 1 and the same alterations in her brother. The subjects displayed a known homozygous disease mutation in the WNT10A gene (p.F228I, rs121908120) and a heterozygous mutation in the EDARADD gene (p.S103F, rs114632254). The frequent p.F228I aminoacid substitution is associated with the autosomal dominant or autosomal recessive form of isolated hypodontia and ectodermal dysplasia (29). The EDARADD mutation $\mathrm{p} . \mathrm{S} 103 \mathrm{~F}$ was previously predicted to be harmful for protein function by bioinformatics analysis (19). This particular combination of genetic variants involving two genes related to teeth development has never been previously described, at least to the best of our knowledge. Both the affected individuals had oligodontia, but individual II-1 had 17 missing teeth and subject II-2 had 6 missing teeth. By WES, we identified the pathogenic mutation in the proband of family 8 that also displays the p.F228I mutation in WNT10A in the homozygous state.
To extend the WES analysis, we focused on families 5, 8 and 9, as genomic DNA was available from all the members with the exception of the unaffected father (I-1) of family 9 . For family 5 , an autosomal recessive form of inheritance seemed most probable. Our analysis identified the PITX2, TTN and MUC16 genes as affected by candidate mutations. The TTN and MUC16 genes were not considered good candidate genes for agenesis prediction, given their high RVIS score, confirming the propensity of these genes to accumulate rare functional mutations with neutral effect (30). Of note, we found a homozygous c.-387delC/G variation in the 5'UTR of the PITX2 gene in the siblings that could be associated with tooth agenesis as we mentioned above. PITX2 is a transcription factor that initiates tooth development, activating amelogenin expression, whose protein product is necessary for enamel formation. Since the mutation is localized in the promoter region of the gene, it may affect its transcriptional activity (31). A more in depth analysis of genetic variants in this family also identified an interesting variant in the BMP4 gene, a non-synonymous SNP in the homozygous state (rs17563; c.T455C:p.V152A) in the affected siblings II-1 and II-2. In a Brazilian study conducted 
Table IV. Selected candidate genes and variants in family 5.

\begin{tabular}{|c|c|c|c|c|c|c|}
\hline Gene.refGene & $\begin{array}{l}\text { ExonicFunc. } \\
\text { refGene }\end{array}$ & Chr & Ref & Alt & Func.refGene & AAChange.refGene \\
\hline \multicolumn{7}{|l|}{ Compound } \\
\hline MUC16 & $\begin{array}{l}\text { Non-synonymous } \\
\text { SNV }\end{array}$ & $\operatorname{chr} 19$ & G & $\mathrm{T}$ & Exonic & MUC16:NM_024690:exon3:c.C12953A:p.A4318D \\
\hline MUC16 & $\begin{array}{l}\text { Non-synonymous } \\
\text { SNV }\end{array}$ & chr19 & $\mathrm{T}$ & $\mathrm{C}$ & Exonic & MUC16:NM_024690:exon3:c.A23897G:p.E7966G \\
\hline MUC16 & $\begin{array}{l}\text { Non-synonymous } \\
\text { SNV }\end{array}$ & $\operatorname{chr} 19$ & $\mathrm{C}$ & $\mathrm{T}$ & Exonic & MUC16:NM_024690:exon12:c.G36581A:p.R12194Q \\
\hline TTN & $\begin{array}{l}\text { Non-synonymous } \\
\text { SNV }\end{array}$ & $\operatorname{chr} 2$ & $\mathrm{C}$ & G & Exonic & $\begin{array}{l}\text { TTN:NM_003319:exon186:c.G75952C:p.E25318Q, } \\
\text { TTN:NM_133432:exon187:c.G76327C:p.E25443Q, } \\
\text { TTN:NM_133437:exon187:c.G76528C:p.E25510Q, } \\
\text { TTN:NM_133378:exon307:c.G95443C:p.E31815Q, } \\
\text { TTN:NM_001256850:exon308:c.G98224C:p.E32742Q, } \\
\text { TTN:NM_001267550:exon358:c.G103147C:p.E34383Q }\end{array}$ \\
\hline TTN & $\begin{array}{l}\text { Non-synonymous } \\
\text { SNV }\end{array}$ & $\operatorname{chr} 2$ & $\mathrm{C}$ & $\mathrm{T}$ & Exonic & $\begin{array}{l}\text { TTN:NM_003319:exon73:c.G18133A:p.D6045N, } \\
\text { TTN:NM_133432:exon74:c.G18508A:p.D6170N, } \\
\text { TTN:NM_133437:exon74:c.G18709A:p.D6237N, } \\
\text { TTN:NM_133378:exon194:c.G37624A:p.D12542N, } \\
\text { TTN:NM_001256850:exon195:c.G40405A:p.D13469N, } \\
\text { TTN:NM_001267550:exon245:c.G45328A:p.D15110N }\end{array}$ \\
\hline TTN & $\begin{array}{l}\text { Non-synonymous } \\
\text { SNV }\end{array}$ & chr2 & $\mathrm{T}$ & $\mathrm{C}$ & Exonic & $\begin{array}{l}\text { TTN:NM_133378:exon90:c.A23131G:p.I7711V, } \\
\text { TTN:NM_001256850:exon91:c.A25912G:p.I8638V, } \\
\text { TTN:NM_001267550:exon93:c.A26863G:p.I8955V }\end{array}$ \\
\hline TTN & $\begin{array}{l}\text { Non-synonymous } \\
\text { SNV }\end{array}$ & chr2 & G & A & Exonic & $\begin{array}{l}\text { TTN:NM_133378:exon77:c.C19445T:p.S6482L, } \\
\text { TTN:NM_001256850:exon78:c.C22226T:p.S7409L, } \\
\text { TTN:NM_001267550:exon80:c.C23177T:p.S7726L }\end{array}$ \\
\hline \multicolumn{7}{|l|}{ Known } \\
\hline PITX2 & UTR5 & chr4 & $\mathrm{C}$ & - & $\begin{array}{l}\text { NM_000325: } \\
\text { c.-387delG }\end{array}$ & \\
\hline BMP4 & Exonic & chr14 & $\mathrm{T}$ & $\mathrm{C}$ & $\begin{array}{l}\text { NM_001202: } \\
\text { c.T455C: } \\
\text { p.V152A }\end{array}$ & \\
\hline
\end{tabular}

on 46 individuals with tooth agenesis and 88 control cases, the CC genotype of BMP4 was more frequent in individuals with 3 or more missing teeth than in the control group $(\mathrm{p}<0.0001)$, leading the authors to the conclusion that this variant was associated with tooth agenesis (32). The same SNP was previously found in two Mexican families with oligodontia (33). Capasso et al (34) observed that the $\mathrm{c} 455 \mathrm{~T}>\mathrm{C}$ substitution altered the BMP4 mRNA secondary structure and that the BMP4 mRNA and protein expression levels were higher for the $\mathrm{T}$ allele in a population in southern Italy with cutaneous melanoma. PAX9 and MSX1 synergistically activate BMP4, and BMP4 is a gene crucial to tooth development as it regulates the passage from bud to cap stages. Since the unaffected parents displayed the same genetic variations of PITX2 and BMP4 in the homozygous state, it was deemed that these variations alone were unlikely to be the direct cause of the observed tooth hypodontia; however, they may still play a role as risk factors or modulators of the phenotype.

Family 8 (a trio with unaffected parents) displayed a probable autosomic recessive form of inheritance. Unfortunately, we were unable to identify a strong candidate gene with a clear role in the physio-pathology of tooth formation and development. Based on RVIS score prioritization criteria, 6 genes emerged affected by functional mutation in this family and not expected to accumulate functional mutation by chance, namely PRR32, PLAC4, nuclear factor 1A (NFIA), tripartite motif containing 14 (TRIM14), carbohydrate sulfotransferase 8 (CHST8) and synaptic Ras GTPase activating protein 1 (SYNGAP1). Of these, 3 genes were unlikely to be involved in tooth agenesis: PLAC4 is mainly involved in placenta tissue formation (35); CHST8 has been associated with autosomal recessive peeling skin syndrome (36); SYNGAP1 is 
Table V. Selected candidate genes and variants in family 8 .

\begin{tabular}{lcccccc}
\hline Chr & Ref & Alt & Func.refGene & Gene.refGene & GeneDetail.refGene & ExonicFunc.refGene \\
\hline $\begin{array}{l}\text { Recessive } \\
\text { chrX }\end{array}$ & G & A & Exonic & ARSH & NA & Non-synonymous SNV \\
$\operatorname{chrX}$ & G & A & Exonic & PRR32 & NA & Non-synonymous SNV \\
$\operatorname{chr} 3$ & C & T & Exonic & TGM4 & NA & Stopgain \\
chr21 & - & 40 bp & Exonic & PLAC4 & NA & Frameshift insertion \\
$\operatorname{chrX}$ & G & A & Exonic & APEX2 & NA & Non-synonymous SNV
\end{tabular}

De novo

$\begin{array}{lccll}\text { chr17 } & 21 \mathrm{bp} & - & \text { Splicing } & \text { MYO19 } \\ \text { chr16 } & \mathrm{T} & \mathrm{G} & \text { Exonic } & \text { IFT140 } \\ \text { chr1 } & \mathrm{A} & \mathrm{C} & \text { Splicing } & \text { NFIA }\end{array}$

NA

NA

Non-synonymous SNV

NM_001145511:exon9:c.12312A >C,

NM_001145512:exon10:c.1390-2A>C,

NM_005595:exon9:c.12552A $>$ C,

NM_001134673:exon9:c.1255-2A>C

$\begin{array}{lllll}\text { chr11 } & - & \text { T } & \text { Exonic } & \text { MUC6 } \\ \text { chr6 } & \text { G } & \text { A } & \text { Exonic } & \text { GPANK1 } \\ \text { chr1 } & \text { G } & \text { C } & \text { Splicing } & \text { NFIA }\end{array}$

\section{NA}

NA

NM_001145511:exon9:c.1231-1G>C,

NM_001145512:exon10:c.1390-1G>C,

NM_005595:exon9:c.12551G>C,

NM_001134673:exon9:c.1255-1G>C

\begin{tabular}{|c|c|c|c|c|}
\hline chr6 & A & G & Exonic & HSPA1L \\
\hline chr11 & $\mathrm{G}$ & A & Exonic & MUC6 \\
\hline chr19 & - & $18 \mathrm{bp}$ & Exonic & RSPH6A \\
\hline $\operatorname{chr} 9$ & $\mathrm{C}$ & $\mathrm{G}$ & Exonic & TRIM14 \\
\hline chr19 & - & AGC & Exonic & CHST8 \\
\hline chr6 & $\begin{array}{l}\text { CACC } \\
\text { ACCA } \\
\text { CCAT }\end{array}$ & - & Exonic & SYNGAF \\
\hline $\operatorname{chrX}$ & $\mathrm{C}$ & $\mathrm{T}$ & Exonic & RBMX \\
\hline chr11 & G & $\mathrm{T}$ & Exonic & MUC6 \\
\hline chr11 & A & G & Exonic & MUC6 \\
\hline chr11 & G & - & Exonic & MUC6 \\
\hline chr1 & $\mathrm{G}$ & - & Splicing & SEC22B \\
\hline chr3 & $\mathrm{C}$ & G & Exonic & IL17RE \\
\hline $\begin{array}{l}\text { Comp } \\
\text { chr2 }\end{array}$ & - & CTGC & Exonic & DNAH7 \\
\hline chr2 & A & $\mathrm{G}$ & Exonic & DNAH7 \\
\hline $\operatorname{chr} 2$ & $\mathrm{C}$ & $\mathrm{T}$ & Exonic & DNAH7 \\
\hline chr11 & - & $\mathrm{T}$ & Exonic & MUC6 \\
\hline chr11 & $\mathrm{G}$ & A & Exonic & MUC6 \\
\hline chr11 & - & TA & Exonic & MUC6 \\
\hline chr11 & $\mathrm{G}$ & $\mathrm{T}$ & Exonic & MUC6 \\
\hline chr11 & $\mathrm{A}$ & $\mathrm{G}$ & Exonic & MUC6 \\
\hline chr11 & $\mathrm{G}$ & - & Exonic & MUC6 \\
\hline chr1 & A & $\mathrm{C}$ & Splicing & NFIA \\
\hline
\end{tabular}

NA
NA
NA
NA
NA

Non-synonymous SNV

Non-synonymous SNV

Non-frameshift insertion

Non-synonymous SNV

Non-frameshift insertion

Non-frameshift insertion

NA
NA
NA
NA

NM_004892:exon6:c.1066-1G>-

NA

Non-synonymous SNV

Non-synonymous SNV

Non-synonymous SNV

Frameshift deletion

NA

Non-synonymous SNV

Frameshift insertion

Non-synonymous SNV

Non-synonymous SNV

Frameshift insertion

Non-synonymous SNV

Frameshift insertion

Non-synonymous SNV

Non-synonymous SNV

Frameshift deletion

NM_001145511:exon9:c.1231-2A>C,

NM_001145512:exon10:c.1390-2A>C, NA 
Table V. Continued.

\begin{tabular}{|c|c|c|c|c|c|c|}
\hline Chr & Ref & Alt & Func.refGene & Gene.refGene & GeneDetail.refGene & ExonicFunc.refGene \\
\hline $\operatorname{chr} 1$ & G & $\mathrm{C}$ & Splicing & NFIA & $\begin{array}{l}\text { NM_001145511:exon9:c.1231-1G>C, } \\
\text { NM_001145512:exon10:c.1390-1G>C, } \\
\text { NM_005595:exon9:c.1255-1G>C, } \\
\text { NM_001134673:exon9:c.1255-1G>C }\end{array}$ & NA \\
\hline $\operatorname{chr} 10$ & A & $\mathrm{T}$ & Splicing & NRAP & $\begin{array}{l}\text { NM_006175:exon38:c.4431+2T>A, } \\
\text { NM_198060:exon39:c.4536+2T>A, } \\
\text { NM_001261463:exon39:c.4536+2T>A }\end{array}$ & NA \\
\hline $\operatorname{chr} 10$ & $\mathrm{~T}$ & G & Exonic & NRAP & NA & Nonsynonymous SNV \\
\hline \multicolumn{7}{|l|}{ Known } \\
\hline PITX2 & UTR5 & chr4 & $\mathrm{C}$ & - & NM_000325:c.-387delG & \\
\hline
\end{tabular}

Table VI. Selected candidate genes and variants in family 9.

\begin{tabular}{|c|c|c|c|c|c|c|c|}
\hline Chr & Ref & Alt & $\begin{array}{l}\text { Func. } \\
\text { refGene }\end{array}$ & $\begin{array}{l}\text { Gene. } \\
\text { refGene }\end{array}$ & $\begin{array}{l}\text { GeneDetail. } \\
\text { refGene }\end{array}$ & $\begin{array}{l}\text { ExonicFunc. } \\
\text { refGene }\end{array}$ & AAChange.refGene \\
\hline \multicolumn{8}{|c|}{ Compound } \\
\hline $\operatorname{chr} 20$ & A & G & Exonic & ANKEF1 & NA & $\begin{array}{l}\text { Non-synonymous } \\
\text { SNV }\end{array}$ & $\begin{array}{l}\text { ANKEF1:NM_198798:exon2:c.A332G:p.D111G, } \\
\text { ANKEF1:NM_022096:exon3:c.A332G:p.D111G }\end{array}$ \\
\hline chr20 & $\mathrm{C}$ & $\mathrm{T}$ & Exonic & COMMD7 & NA & $\begin{array}{l}\text { Non-synonymous } \\
\text { SNV }\end{array}$ & $\begin{array}{l}\text { COMMD7:NM_001099339:exon1:c.G5A:p.G2D, } \\
\text { COMMD7:NM_053041:exon1:c.G5A:p.G2D }\end{array}$ \\
\hline chr14 & $\mathrm{T}$ & $\mathrm{C}$ & Exonic & DDHD1 & NA & $\begin{array}{l}\text { Non-synonymous } \\
\text { SNV }\end{array}$ & $\begin{array}{l}\text { DDHD1:NM_001160148:exon6:c.A1411G:p.I471V, } \\
\text { DDHD1:NM_030637:exon6:c.A1411G:p.I471V, } \\
\text { DDHD1:NM_001160147:exon7:c.A1432G:p.I478V }\end{array}$ \\
\hline chr8 & A & $\mathrm{G}$ & Exonic & EBF2 & NA & $\begin{array}{l}\text { Non-synonymous } \\
\text { SNV }\end{array}$ & EBF2:NM_022659:exon7:c.T560C:p.L187S \\
\hline chr17 & $\mathrm{G}$ & $\mathrm{T}$ & Exonic & EVPL & NA & $\begin{array}{l}\text { Non-synonymous } \\
\text { SNV }\end{array}$ & EVPL:NM_001988:exon11:c.C1213A:p.L405M \\
\hline $\operatorname{chr} 3$ & $\mathrm{C}$ & $\mathrm{T}$ & Exonic & SUCLG2 & NA & $\begin{array}{l}\text { Non-synonymous } \\
\text { SNV }\end{array}$ & $\begin{array}{l}\text { SUCLG2:NM_001177599:exon10:c.G1124A:p.G375E, } \\
\text { SUCLG2:NM_003848:exon10:c.G1124A:p.G375E }\end{array}$ \\
\hline $\operatorname{chr} 3$ & $\mathrm{C}$ & $\mathrm{T}$ & Exonic & SUCLG2 & NA & $\begin{array}{l}\text { Non-synonymous } \\
\text { SNV }\end{array}$ & $\begin{array}{l}\text { SUCLG2:NM_001177599:exon10:c.G1123A:p.G375R, } \\
\text { SUCLG2:NM_003848:exon10:c.G1123A:p.G375R }\end{array}$ \\
\hline $\operatorname{chr} 17$ & $\mathrm{~T}$ & $\mathrm{C}$ & Exonic & SUPT6H & NA & $\begin{array}{l}\text { Non-synonymous } \\
\text { SNV }\end{array}$ & SUPT6H:NM_003170:exon32:c.T4393C:p.C1465R \\
\hline \multicolumn{8}{|c|}{ De novo } \\
\hline $\operatorname{chr} 3$ & $\mathrm{C}$ & $\mathrm{T}$ & Exonic & SUCLG2 & NA & $\begin{array}{l}\text { Non-synonymous } \\
\text { SNV }\end{array}$ & $\begin{array}{l}\text { SUCLG2:NM_001177599:exon10:c.G1124A:p.G375E, } \\
\text { SUCLG2:NM_003848:exon10:c.G1124A:p.G375E }\end{array}$ \\
\hline chr3 & $\mathrm{C}$ & $\mathrm{T}$ & Exonic & SUCLG2 & NA & $\begin{array}{l}\text { Non-synonymous } \\
\text { SNV }\end{array}$ & $\begin{array}{l}\text { SUCLG2:NM_001177599:exon10:c.G1123A:p.G375R, } \\
\text { SUCLG2:NM_003848:exon10:c.G1123A:p.G375R }\end{array}$ \\
\hline
\end{tabular}

responsible for non-syndromic mental retardation autosomal dominant form 5 (OMIM 612621) (37), a phenotype not reported in this family. The PRR32 gene is poorly characterized and difficult to evaluate. NFIA and TRIM14 are two transcription factor genes and they may represent good candidates for further studies aimed at assessing their involvement in the regulatory network driving teeth morphogenesis. NFIA may be of particular interest, since mutations in this gene cause craniofacial abnormalities in mice and craniosynostosis in humans (38). However, homozygous null mutations in the gene have been associated with kidney, nervous and fertility phenotypes that were not observed in this family. Of note, this family was also a carrier of the genetic variant c.387delC/G in the 5'UTR of PITX2 (found in the homozygous state in the 
proband and in his unaffected brother and in the heterozygous state in their unaffected mother in family 5; discussed above), suggesting that a complex interplay of genetic risk factors may be involved in this disease.

Finally, family 9 included a pair of monozygotic twins with hypodontia and the recessive inheritance model was likely. Unfortunately, DNA for the father was not available for the study. Among the genetic variants found, only the one in COMMD7 may have a link with the disease. COMMD7 is in fact a NEMO interacting protein involved in the termination of $N F-\kappa B$ signaling that is activated by the EDA-EDAR-EDARADD complex (39). This mutation is predicted to be damaging with a score of 1 by PolyPhen-2. Further studies are necessary to establish whether this heterozygous mutation in the proband twin may be involved in the pathological phenotype.

In conclusion, in this study, using WES analysis, we identified the causative mutations in cases with a more severe phenotype (families 1 and 7, where 17 and 16 teeth were missing, respectively). We did not immediately identify variants that may be disease-causing for families with a light phenotype, such as families 5 and 9 (maximum 4 missing teeth) and with a mild oligodontia, such as family 8 (6 missing teeth). However, our analysis of these families highlighted some interesting candidate genes that may be considered targets of future functional studies. However, we cannot exclude that the manifestation of the phenotypic traits may also depend or be modulated by other mechanisms, including long and small noncoding RNAs (i.e., miRNAs), epigenetic modifications, such as DNA methylation and histone modifications or regulatory DNA variants. Finally, since a complex regulatory network contributes to tooth formation with multitudes of genes potentially involved, we consider that WES may be an effective strategy with which to detect the genetic defects related to tooth agenesis, particularly in severe cases of oligodontia in sporadic and familial cases. The determination of the genetic causes of tooth agenesis is important for genetic counseling and for anticipating the problems related to the clinical management of dental anomalies, in order to ensure a correct occlusion, particularly during developing dentition in children.

\section{Acknowledgements}

We warmly thank Dr M. Crosatti (University of Leicester, UK) for the linguistic revision of the manuscript. This study was supported by Ministero dell'Istruzione, dell'Università e della Ricerca (MIUR) local funds of the University of Brescia. Publication costs were covered by Grant 'New Opportunities and Ways towards ERC' (NOW ERC, Project: 2014-2256) from Fondazione Cariplo and Regione Lombardia.

\section{References}

1. Bozga A, Stanciu RP and Mănuc D: A study of prevalence and distribution of tooth agenesis. J Med Life 7: 551-554, 2014.

2. Brook AH, Jernvall J, Smith RN, Hughes TE and Townsend GC: The dentition: The outcomes of morphogenesis leading to variations of tooth number, size and shape. Aust Dent J 59 (Suppl 1): 131-142, 2014

3. Brook AH: Multilevel complex interactions between genetic, epigenetic and environmental factors in the aetiology of anomalies of dental development. Arch Oral Biol 54 (Suppl 1): S3-S17, 2009.
4. Cobourne MT: Familial human hypodontia - is it all in the genes? Br Dent J 203: 203-208, 2007.

5. Yin W and Bian Z: The Gene Network Underlying Hypodontia. J Dent Res 94: 878-885, 2015.

6. Zhang YD, Chen Z, Song YQ, Liu C and Chen YP: Making a tooth: Growth factors, transcription factors, and stem cells. Cell Res 15: 301-316, 2005.

7. Feng C, Xu Z, Li Z, Zhang D, Liu Q and Lu L: Down-regulation of Wnt10a by RNA interference inhibits proliferation and promotes apoptosis in mouse embryonic palatal mesenchymal cells through Wnt $/ \beta$-catenin signaling pathway. J Physiol Biochem 69: 855-863, 2013.

8. Nassif A, Senussi I, Meary F, Loiodice S, Hotton D, Robert B, Bensidhoum M, Berdal A and Babajko S: Msx1 role in craniofacial bone morphogenesis. Bone 66: 96-104, 2014.

9. Ogawa T, Kapadia H, Feng JQ, Raghow R, Peters H and D'Souza RN: Functional consequences of interactions between Pax9 and Msx1 genes in normal and abnormal tooth development. J Biol Chem 281: 18363-18369, 2006.

10. Suryadeva S and Khan MB: Role of homeobox genes in tooth morphogenesis: A review. J Clin Diagn Res 9: ZE09-ZE12, 2015.

11. Lammi L, Arte S, Somer M, Jarvinen H, Lahermo P, Thesleff I, Pirinen $S$ and Nieminen P: Mutations in AXIN2 cause familial tooth agenesis and predispose to colorectal cancer. Am J Hum Genet 74: 1043-1050, 2004.

12. Galluccio G, Castellano M and La Monaca C: Genetic basis of non-syndromic anomalies of human tooth number. Arch Oral Biol 57: 918-930, 2012.

13. Song S, Zhao R, He H, Zhang J, Feng H and Lin L: WNT10A variants are associated with non-syndromic tooth agenesis in the general population. Hum Genet 133: 117-124, 2014.

14. Arzoo PS, Klar J, Bergendal B, Norderyd J and Dahl N: WNT10A mutations account for $1 / 4$ of population-based isolated oligodontia and show phenotypic correlations. Am J Med Genet A 164A: 353-359, 2014.

15. Nikopensius T, Annilo T, Jagomägi T, Gilissen C, Kals M, Krjutškov K, Mägi R, Eelmets M, Gerst-Talas U, Remm M, et al: Non-syndromic tooth agenesis associated with a nonsense mutation in ectodysplasin-A (EDA). J Dent Res 92: 507-511, 2013.

16. Klein ML, Nieminen P, Lammi L, Niebuhr E and Kreiborg S: Novel mutation of the initiation codon of PAX9 causes oligodontia. J Dent Res 84: 43-47, 2005.

17. De Muynck S, Schollen E, Matthijs G, Verdonck A, Devriendt K and Carels C: A novel MSX1 mutation in hypodontia. Am J Med Genet A 128A: 401-403, 2004.

18. Kim JW, Simmer JP, Lin BP and Hu JC: Novel MSX1 frameshift causes autosomal-dominant oligodontia. J Dent Res 85: 267-271, 2006.

19. Arte S, Parmanen S, Pirinen S, Alaluusua $S$ and Nieminen P: Candidate gene analysis of tooth agenesis identifies novel mutations in six genes and suggests significant role for WNT and EDA signaling and allele combinations. PLoS One 8: e73705, 2013.

20. Li H and Durbin R: Fast and accurate long-read alignment with Burrows-Wheeler transform. Bioinformatics 26: 589-595, 2010.

21. Van der Auwera GA, Carneiro MO, Hartl C, Poplin R, Del Angel G, Levy-Moonshine A, Jordan T, Shakir K, Roazen D, Thibault J, et al: From FastQ data to high confidence variant calls: the Genome Analysis Toolkit best practices pipeline. Curr Protoc Bioinformatics 43: 1110 11-33, 2013.

22. Wang K, Li M and Hakonarson H: ANNOVAR: Functional annotation of genetic variants from high-throughput sequencing data. Nucleic Acids Res 38: e164, 2010.

23. Landrum MJ, Lee JM, Benson M, Brown G, Chao C, Chitipiralla S, Gu B, Hart J, Hoffman D, Hoover J, et al: ClinVar: Public archive of interpretations of clinically relevant variants. Nucleic Acids Res 44: D862-D868, 2016.

24. Petrovski S, Wang Q, Heinzen EL, Allen AS and Goldstein DB: Genic intolerance to functional variation and the interpretation of personal genomes. PLoS Genet 9: e1003709, 2013.

25. Adzhubei IA, Schmidt S, Peshkin L, Ramensky VE, Gerasimova A, Bork P, Kondrashov AS and Sunyaev SR: A method and server for predicting damaging missense mutations. Nat Methods 7: 248-249, 2010.

26. Shihab HA, Gough J, Cooper DN, Stenson PD, Barker GL, Edwards KJ, Day IN and Gaunt TR: Predicting the functional, molecular, and phenotypic consequences of amino acid substitutions using hidden Markov models. Hum Mutat 34: 57-65, 2013. 
27. Vieira AR, Meira R, Modesto A and Murray JC: MSX1, PAX9, and TGFA contribute to tooth agenesis in humans. J Dent Res 83: 723-727, 2004

28. Tallón-Walton V, Manzanares-Céspedes MC, Carvalho-Lobato P, Valdivia-Gandur I, Arte S and Nieminen P: Exclusion of PAX and MSX1 mutation in six families affected by tooth agenesis. A genetic study and literature review. Med Oral Patol Oral Cir Bucal 19: e248-e254, 2014

29. van den Boogaard MJ, Créton M, Bronkhorst Y, van der Hout A, Hennekam E, Lindhout D, Cune M and Ploos van Amstel HK: Mutations in WNT10A are present in more than half of isolated hypodontia cases. J Med Genet 49: 327-331, 2012.

30. MacArthur DG, Manolio TA, Dimmock DP, Rehm HL, Shendure J, Abecasis GR, Adams DR, Altman RB, Antonarakis SE, Ashley EA, et al: Guidelines for investigating causality of sequence variants in human disease. Nature 508: 469-476, 2014.

31. Lubitz SA, Sinner MF, Lunetta KL, Makino S, Pfeufer A, Rahman R, Veltman CE, Barnard J, Bis JC, Danik SP, et al: Independent susceptibility markers for atrial fibrillation on chromosome 4q25. Circulation 122: 976-984, 2010.

32. Antunes LS, Küchler EC, Tannure PN, Lotsch PF, Costa MC, Gouvêa CV, Olej B and Granjeiro JM: TGFB3 and BMP4 polymorphism are associated with isolated tooth agenesis. Acta Odontol Scand 70: 202-206, 2012

33. Mu Y, Xu Z, Contreras CI, McDaniel JS, Donly KJ and Chen S: Phenotype characterization and sequence analysis of BMP2 and BMP4 variants in two Mexican families with oligodontia. Genet Mol Res 11: 4110-4120, 2012.
34. Capasso M, Ayala F, Russo R, Avvisati RA, Asci R and Iolascon A: A predicted functional single-nucleotide polymorphism of bone morphogenetic protein- 4 gene affects mRNA expression and shows a significant association with cutaneous melanoma in Southern Italian population. J Cancer Res Clin Oncol 135: 1799-1807, 2009.

35. Yang L, Sun HY, Chen DZ, Lu MD, Tang Y and Xiao JP: Explore the dynamic alternation of gene PLAC4 mRNA expression levels in maternal plasma in second trimester for nonivasive detection of trisomy 21. Obstet Gynecol Sci 58: 261-267, 2015.

36. Cabral RM, Kurban M, Wajid M, Shimomura Y, Petukhova L and Christiano AM: Whole-exome sequencing in a single proband reveals a mutation in the CHST8 gene in autosomal recessive peeling skin syndrome. Genomics 99: 202-208, 2012.

37. Parker MJ, Fryer AE, Shears DJ, Lachlan KL, McKee SA, Magee AC, Mohammed S, Vasudevan PC, Park SM, Benoit V, et al: De novo, heterozygous, loss-of-function mutations in SYNGAP1 cause a syndromic form of intellectual disability. Am J Med Genet A 167A: 2231-2237, 2015.

38. Nyboe D, Kreiborg S, Kirchhoff M and Hove HB: Familial craniosynostosis associated with a microdeletion involving the NFIA gene. Clin Dysmorphol 24: 109-112, 2015.

39. Esposito E, Napolitano G, Pescatore A, Calculli G, Incoronato MR, Leonardi A and Ursini MV: COMMD7 as a novel NEMO interacting protein involved in the termination of NF- $\kappa \mathrm{B}$ signaling. J Cell Physiol 231: 152-161, 2016. 